\title{
Design and Implementation of a Reliable and Environmental Friendly Smart House System
}

\author{
Diego Paucar ${ }^{1}$, Nida Imtiaz ${ }^{2}$ and Mihaela $\operatorname{Radu}^{3^{*}}$ \\ ${ }^{1}$ Broadband Office Solutions, Great Neck, NY, USA \\ ${ }^{2}$ Nasdaq, Philadelphia, PA, USA \\ ${ }^{3}$ State University of New York, NY, USA \\ Farmingdale State College \\ 1'dpaucar@bbosolutions.com, 2Nida.Imtiaz@nasdaq.com, \\ ${ }^{3}$ radum@farmingdale.edu
}

\begin{abstract}
With the advance of technologies, design and development of smart houses is becoming a very important area of research applications. The expectations of smart house users are a more rational use of resources, greater security and safety, and increased comfort. In this paper the design and implementation of a reliable and environmental-friendly smart home system is presented. The research project focuses on energy savings, security, safety and increased comfort for the users. The human interaction with the system is achieved through intelligent and user's friendly interfaces, allowing both local and remote control of the house. The entire system is built around an inexpensive microcontroller platform, the Arduino platform, using real sensors and actuators. The local control is achieved through a keypad and an LCD display, while the remote control includes an Arduino Ethernet based micro web-server, and an Android based app, which can be used from any Android supported device. The system is affordable, user friendly and easy to adapt, allowing to add new devices and functions, without altering previously built functions. All the functions of the smart house were tested and are fully functional, proving the feasibility and the effectiveness of the design. A reliability analysis was also performed, using industrial grade CAD tools. Estimating the reliability of various blocks of the system and identifying components based on their failure rates, help the designer to make the necessary changes to the design, choosing more reliable components, incorporating spares or adding fault tolerant features.
\end{abstract}

Keywords: smart house, energy, safety, security, sensors, microcontroller, remote control, smart phone, reliability analysis

\section{Introduction}

With the advance of technologies, design and development of smart houses is becoming a very important area of research applications. The expectations of smart house users are a more rational use of resources, greater safety and security, and increased comfort. The need for smart homes has increased in recent years, due to increases in energy bills, home security and safety concerns.

A smart house offers also powerful means for helping and supporting special needs of the elderly and people with disabilities [1]. Considering all the possible inhabitants living in in a smart home, from infants to elderly people, designing a house that operates in a reliable and safe manner is crucial A large number of applications in a smart home are controlled by smart devices that incorporates various sensors. Several incidents with smart thermostats, fans, and smoke and carbon monoxide alarms highlight the need for

* Corresponding Author 
smart devices to operate reliable. The information collected by various sensors is transmitted in some cases over the Internet (Internet-of-Things). Recent consumer surveys show that people are concerned about privacy and security regarding personal information, but also the vulnerabilities found in home appliances [2]. Estimating the reliability of various hardware blocks of the smart house system and identifying components based on their failure rates, helps the designer to make the necessary changes, choosing more reliable components, incorporating spares or adding fault-tolerant features.

Saving electrical energy, gas, water, and other natural resources affects the global environment in a positive way. Preventing fires, smoke and gas leakage avoids polluting the atmosphere. Security systems prevent unwanted intruders, who may damage the property and possibly endanger the environment through malice fires, water floods, etc.

There are several factors that need to be considered when designing a reliable and environmental-friendly smart house. The system should be affordable, user friendly and easy to adapt, in order to incorporate new devices and features [1]. Current research in the area of smart house covers usually areas such as: (i) smart home design, covering a broad spectrum of functions from the efficient use of resources to the increased comfort for the inhabitants, offering powerful means for helping and supporting vulnerable people; (ii) authentication and security, from biometrics to sophisticated protected access into the house; (iii) safety, from alarm systems monitoring gas leakage, smoke, fire, to failure mode, safety and reliability analysis/estimation of sensors and actuators, which represent an integral part of a smart house system; (iv) human interaction, developing intelligent and user friendly interfaces between the user and the house system. The advance of IoT technology (Internet of Things) allows the designers to come with creative and intelligent interfaces at an advanced pace, offering new ideas and a broad space for development. Smart phones, tablets can be used to control a variety of devices which are connected to the smart house system [3].

In this paper the design and implementation of an environmental friendly and reliable smart home system is presented. The research project focuses on: energy savings, security safety and increased comfort for the users. The human interaction with the system is achieved through intelligent and user's friendly interfaces, allowing both local and remote control of the house. The entire system is built around an inexpensive microcontroller platform, the Arduino platform, using real sensors and actuators. The local control is achieved through a keypad and a LCD display, while the remote control includes an Arduino Ethernet based micro web-server, and an Android based app, which can be used from any Android supported device. The system is affordable, user friendly and easy to adapt, allowing to add new devices and functions, without altering previously built functions. All the functions of the smart house were tested and are fully functional, proving the feasibility and effectiveness of the design. A reliability analysis was also performed, using industrial grade CAD tools.

While the majority of the research papers dedicated to smart house design, which will be discussed in the next section of this paper, focusses on one or two of the research areas previously enumerated, the smart house system presented in this paper implements creative functions covering several areas of smart house research using modern, inexpensive and reliable technologies. The prototype of the smart house is currently used for live demonstration emulating customer-owned equipment and to raise public awareness regarding the advantages of a smart house system.

The rest of the paper is organized as follows. Section 2 is an overview of the research in the areas of smart house design. Section 3 presents the functions of the environmental friendly and reliable smart house system. Section 4 presents the hardware and software implementation of the smart house. Section 5 presents the tests and results of the testing performed on the smart house. Section 6 presents reliability analysis of the smart house, while Section 7 presents conclusions and future work. 


\section{Similar Research}

There is a rich body of research papers dedicated to the design and development of smart houses. The majority of the research papers dedicated to smart house focusses on one or two of the research areas previously enumerated, presenting fully or partially implemented projects as work-in-progress.

In the area of smart home design, a large number of research papers focusses on energy savings. Reference [3] presents the prototype of a smart home, built around PIC18F458 microcontroller, allowing the control of lights, air conditioning and fans. Reference [4] presents a low-cost and flexible solution to control and monitor home appliances using Smart Plug devices and PIC32MX340512H microcontroller. Users can remotely switch power on/off, get information on the device's power consumption, and control the ambient temperature. Reference [5] presents an Arduino based remote control system aiming to save power, but also to improve the security and protection of the house.

To increase the users' comfort reference [6] proposes a system based on mobile sensing that does not require user's intervention. Mobile sensing is used to record daily activities of the user and automatically gives response to the user. By using mobile sensing, the design of the smart house can be optimized for a specific user. Reference [7] presents an ubiquitous collaboration system for home activities in the a smart home. It provides services for family members to support their individual or collaborative activities.

In the area of authentication and security, reference [8] focuses on the home security by proposing two systems, one based on GSM technology and the other using a web camera to detect intruders. The system uses an ATMEGA 644p microcontroller and GSM_Gps Module (sim548c). Reference [9] presents a similar home security project, based on ATMEGA16 microcontroller and GSM technology. Reference [10] proposes a home security system based on a Rasberry pi Microcontroller and an Android phone monitoring home space to detect intruders and visitors. Reference [11] presents a door locks automation system using Bluetooth-based Android Smartphone. The system is designed to simulate an electronic key, which is controlled through a Bluetooth on a smart phone and other mobile devices. Reference [12] presents a similar project, allowing the user to lock and unlock a door from inside or outside a house using a wireless system. Reference [13] proposes an ear biometrics which considers both shape and texture information to represent ear's image during ear's recognition authentication.

In the area of safety, reference [14] presents a system that detects smoke and give alerts to the users via SMS together with the sound of the alarm, using an Arduino microcontroller. Reference [15] focusses on failure analysis methods for sensors, while reference [16] presents reliability analysis for temperature sensors and data acquisition system based on microcontrollers, which are important blocks in a smart house application.

Safety is essential for any engineering system, especially for systems used by vulnerable people, such as people with disabilities, and older people. Systems are required to be robust and reliable as the person with disabilities will rely on the installed devices [17-18]. Reference [19] explores the potential pitfalls and actual problems with the use of smart home technology for vulnerable people, trying to answer questions such as how can technology be made safer and more reliable within the framework of Custodian software development.

In the area of (iv) human interaction, researchers take full advantage of the advance of technologies in the areas of wireless communication, Internet of Things, smart phones etc. With the dramatic increase in smart phone users, smart phones have gradually turned into an all purpose portable device, used for everyday life activities [1]. References [1,3-12,14] present different versions of smart house control using smart phone, associated apps and GUI to control and monitor the proposed smart house project. Reference [1] presents a 
smart house project aiming to all areas of research, but it does not present the final prototype of the house.

\section{Main Functions of the Smart House System}

The main functions of the Smart House are presented in Figure 1. The entire system is built around an inexpensive microcontroller platform, the Arduino platform, using real sensors and actuators. The local control is achieved through a keypad and an LCD display, while the remote control includes an Arduino Ethernet based micro web-server, and a smartphone application, which can be used from any device supported by Android Operating System and iPhone Operating System (iOS).
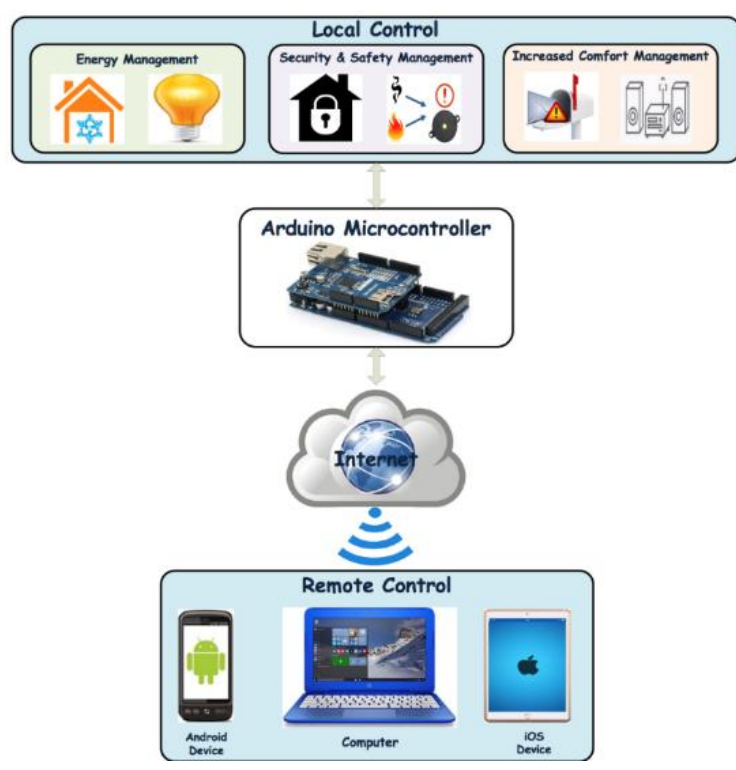

Figure 1. Smart House System

\subsection{Energy Management Function}

The energy management function of the smart house includes two subsystems, the light control subsystem and the air conditioning subsystem, both trying to minimize the energy consumption in the house.

The light control subsystem monitors the status of the lights in different areas of the house, and detects human presence in those areas. If the system detects a light turned on and there is no motion detected the light will be turned off after five minutes.

The air conditioning subsystem monitors the temperature and humidity in the house and control the fan's speed to maintain a desired temperature constant. This subsystem also checks continuously the windows' status (open or closed), and reduces the fan speed by $50 \%$, when a window is detected open. Figure 2 presents the air conditioning subsystem. 


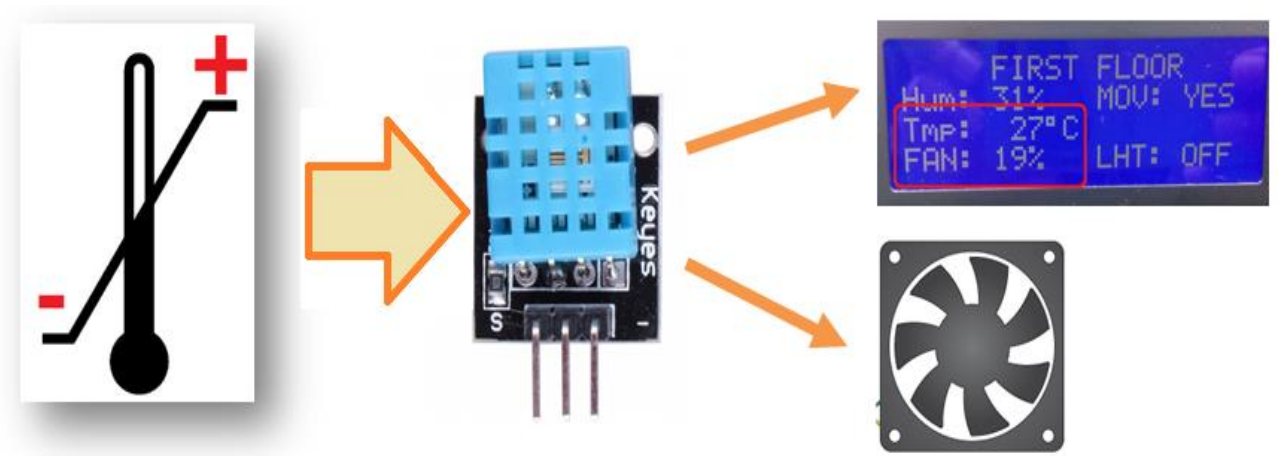

Figure 2. Air Conditioning Subsystem

\subsection{Security Function}

The security function is implemented through a subsystem that controls the house's entrance door locks (main door and balcony door). This subsystem has three security levels: "Total Locked", "Partially Locked", and "Unlocked". For the "Total Locked" level, all the doors will be locked simultaneously, and the alarm will be triggered in case movement is detected. For the "Partially Locked" level, all the doors will be locked simultaneously, but the user will be allowed to move around the house without triggering the alarm. For the "Unlocked" level, all the doors will be unlocked and the alarm will be deactivated. To unlock the house, the user has to enter the correct PIN. The alarm will be triggered every time the incorrect PIN is entered.

\subsection{Safety Function}

The safety function is implemented through a subsystem using asensor that detects fire, smoke and gas leakage. If any of these hazards are detected in the house, then the alarm will be triggered as presented in Figure 3. To help people with hearing disability the emergency lights located inside and outside the house will start blinking.

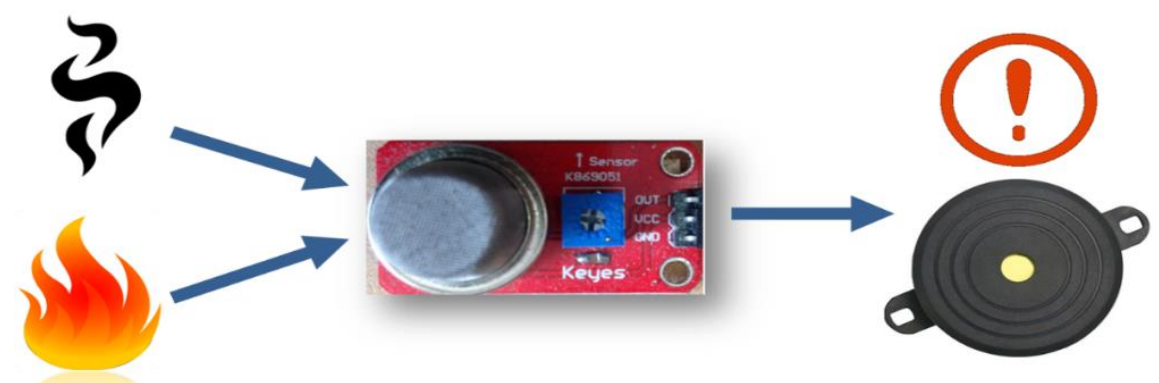

Figure 3. Safety Subsystem - Fire and Smoke Detector

\subsection{Increased User's Comfort Function}

One of the key aspects of designing the smart house is to increase inhabitants' comfort. This function is implemented through the mailbox, backyard lighting, music, and remotely controlled garage door subsystems.

The mailbox subsystem notifies the user if mail is delivered. The purpose of this subsystem is to avoid unnecessary trips to the mail box.

The backyard lighting subsystem turns off the light in the backyard when no movement is detected at night. A motion sensor and a photo resistor sensor are used to control the backyard light. Figure 4 presents the backyard lighting subsystem. 


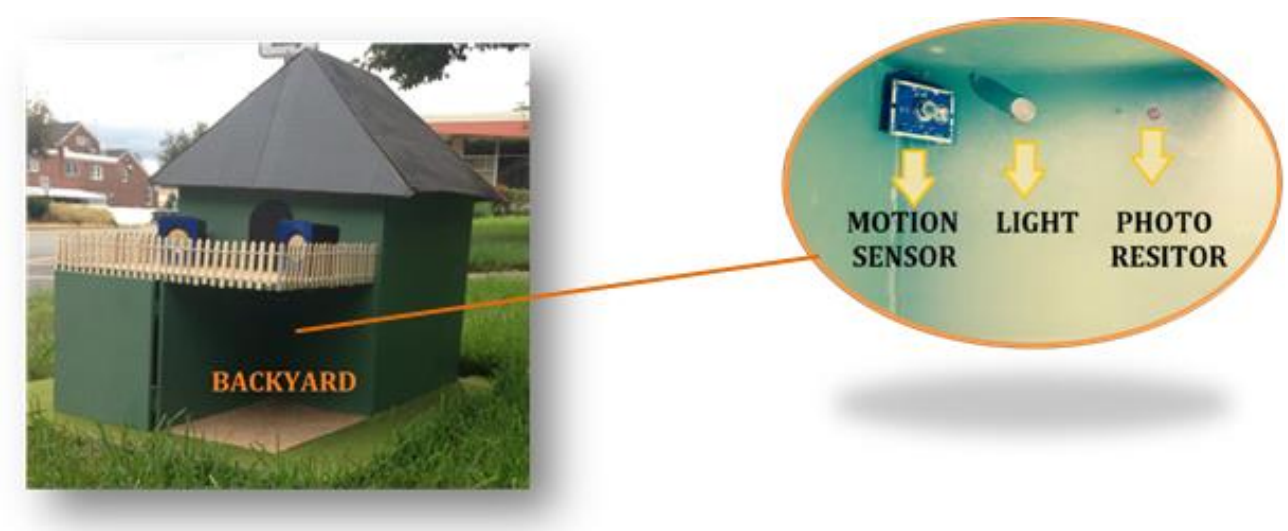

Figure 4. Backyard Light Subsystem

The music subsystem allows the user to listen music throughout the house, as presented in Figure 5. It uses an MP3 shield that has an SD card and an auxiliary port. The MP3 shield reads the audio files from the SD card port and sends the audio signal to the auxiliary port. The MP3 shield is compatible with any audio player with an auxiliary port.

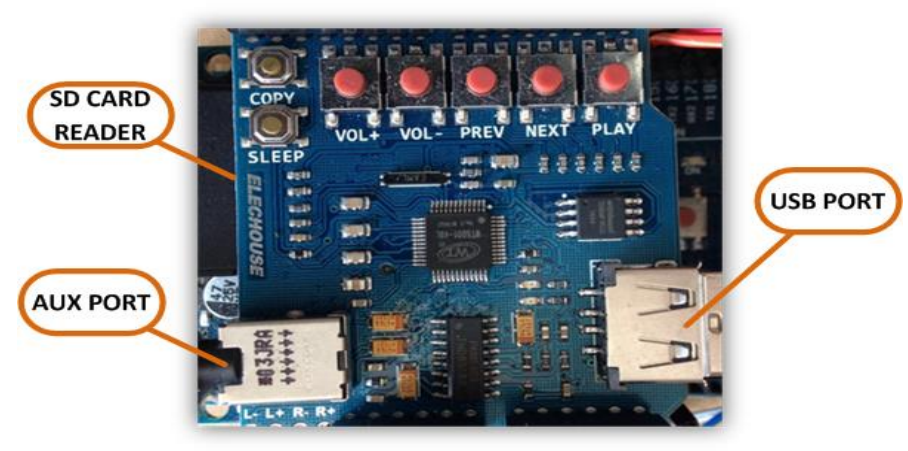

Figure 5. Music Subsystem

The garage door subsystem is designed for the user's convenience. The garage door can be opened and closed remotely from a smartphone, tablet or PC. The user can use the smartphone and open the garage door, instead of opening it manually or using the garage door remote control device, which is usually a bulky and unreliable device. Figure 6 presents the garage door subsystem.

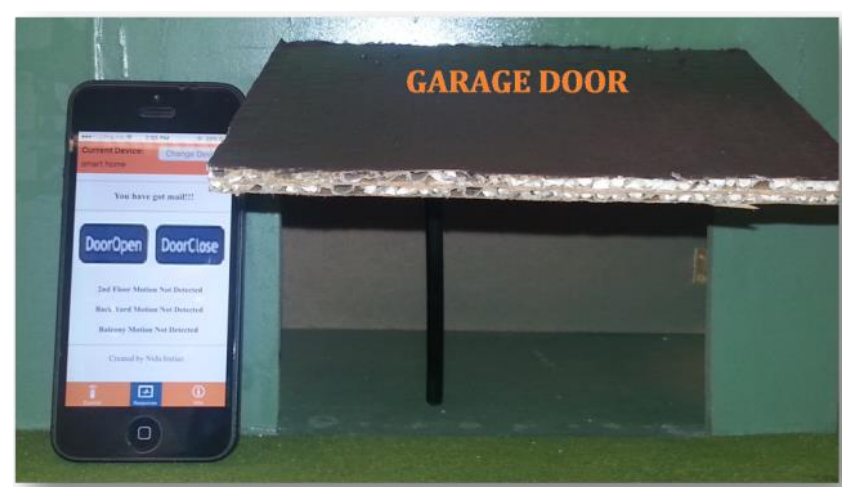

Figure 6. Garage Door Subsystem 


\subsection{Control of the House}

The house's functions can be controlled locally or remotely, as seen in Figure 7.

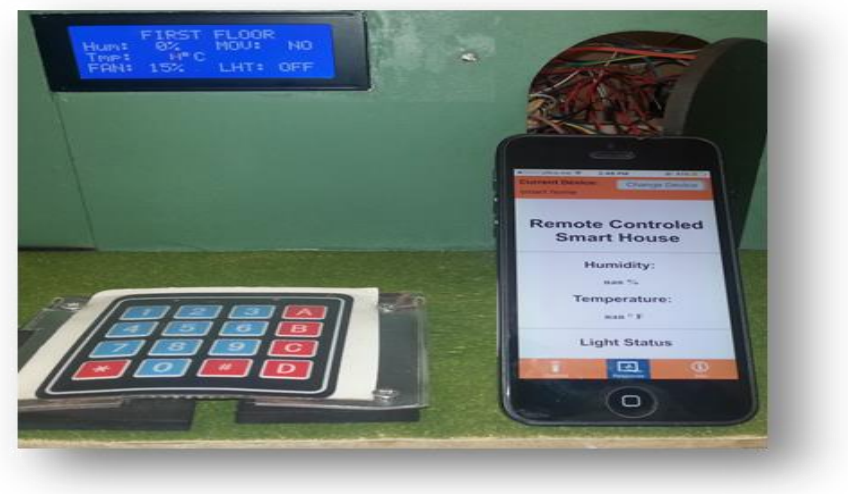

\section{Figure 7. Locally and Remotely Control of the House}

For local control, a display (LCD screen) and a keypad are the user's interactive interface, as presented in Figure 8. The LCD shows a menu indicating different areas of the house that are being monitored by the subsystem such as: first floor, second floor, balcony and garage. Every area of the house is allocated a sub-menu that displays the temperature, humidity, movement, light status, and fan speed for that specific location (rooms, garage, etc.). Each mentioned area has a character of the keypad assigned to it. In order to access a particular area, the user has to use the keypad and select that specific area.

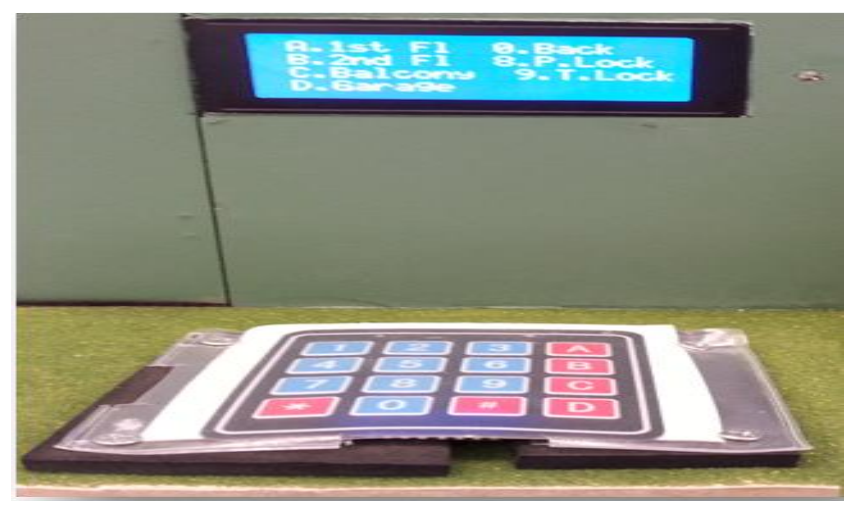

Figure 8. Local Control of the House

For remote control, the Arduino microcontroller uses an Arduino Ethernet shield module that behaves as an embedded server. A web graphical user interface was developed to allow remote control of different functions of the house. The GUI (Graphical User Interface) displays different areas of the house monitored by the subsystems previously mentioned. It displays temperature, movement, humidity, etc. The GUI has action buttons, allowing the user to turn on/off the lights, open the garage door, check the mail, etc. The web graphical user interface is presented in Figure 9. To access the web GUI using an Android/iOS device with internet connection, the user has to download the free smartphone application called "Remote Control House". To synchronize the Android/iOS device with the Arduino Ethernet shield, the Arduino Ethernet Shield's associated IP address is used. The user can also access the web GUI by using the internet browser and entering the Arduino Ethernet Shield's associated IP address as a URL [20-21]. 


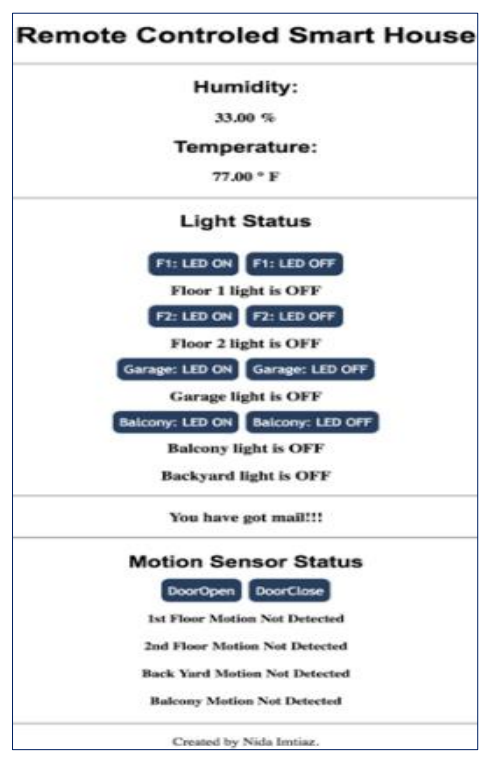

Figure 9. User Interface

3.5.1. Control of the Energy Management Function: All the lights of the house can be controlled remotely using the web GUI. The user has to select the appropriate action button to turn on or off a light, as presented in Figure 9. Since the web GUI is updated every other second, the user can check the status of the lights. The air conditioning subsystem can be controlled locally using the interactive interface. To check the performance of the air conditioning system, the user has to select the desired room as presented in Figures 7 and 8. If a window is open when the fan is operating, a light located next to the interactive interface will start blinking alerting the user.

3.5.2. Control of the Security Function: The security levels of the house can be controlled from the interactive interface. To select any of these options the user has to use the keypad by entering number 8 or 9, as presented in Figure 10. To change to a different level of security, the user has to disable the current one by entering the PIN ****\# as presented in Figure 10. The alarm will be activated every time a wrong PIN is prompted. The security level cannot be controlled remotely to avoid security issues. Every time a level of security is disabled, the security function sets the security level to "Unlocked" by default.
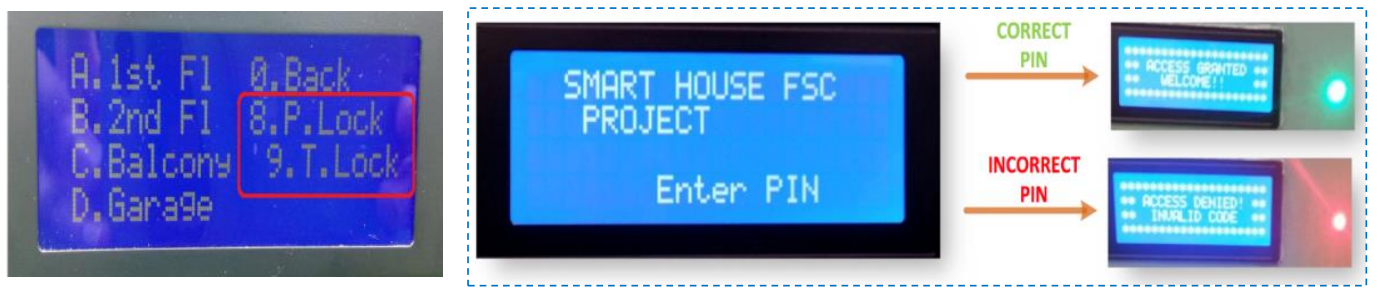

Figure 10. Security Levels and Pin for the House

3.5.3. Control of the Safety Function: The fire and smoke detector located inside the house activates the alarm (Buzzer) and triggers the alert light when a smoke or fire is present in the air, so the user can take the appropriate action.

3.5.4. Control of the Increased User's Comfort Function: The mailbox box subsystem can be monitored continuously. When mail is detected, the user is alerted by the "mailbox light" located inside the house as presented in Figures 11 and 12. 


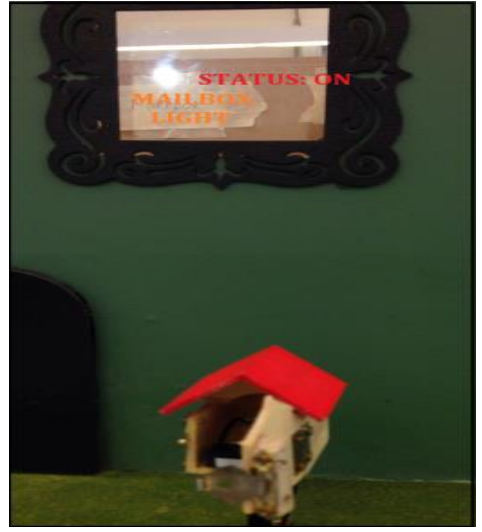

Figure 11. Mailbox Light- No Mail

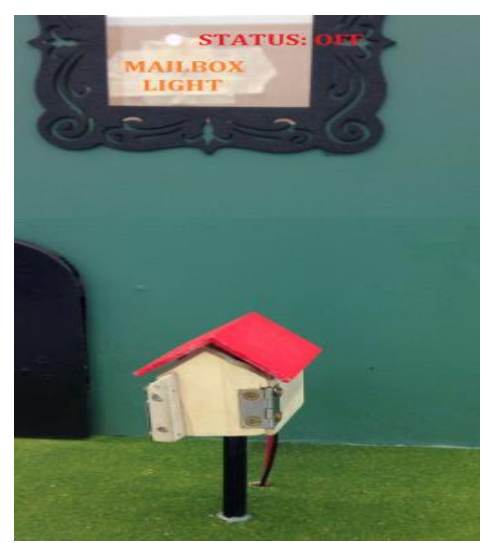

Figure 12. Mailbox Light- Mail detected

The mailbox subsystem can be controlled remotely. To check if mail was delivered, the user has to login to the web GUI to check the mailbox status. If the mail is detected, the mailbox status will display "You have got mail!", as presented in Figure 13.

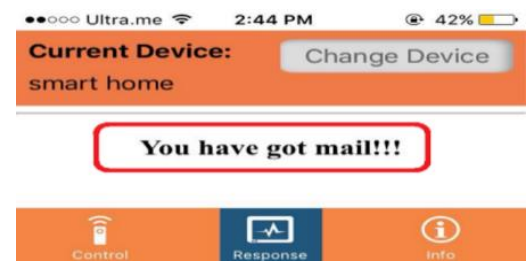

Figure 13. Remote Control of the Mail Subsystem

The backyard lighting subsystem can be monitored remotely. The subsystem continuously updates the backyard light status and the status of the motion detector on the web GUI. The user can check from anywhere in the world if there is an unexpected intruder present in the backyard as presented in Figure 14.

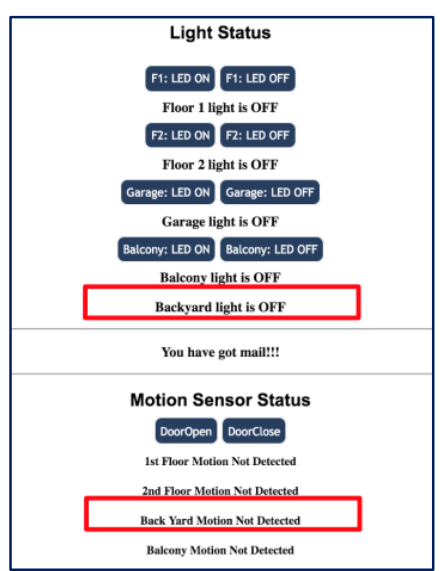

Figure 14. Backyard Light Control using Web-server

The Music subsystem can be controlled locally. The user can play and control audio files read from the SD card through the Board buttons such Play, Pause, Volume Up and Down, and Sleep, as presented in Figure 15. 


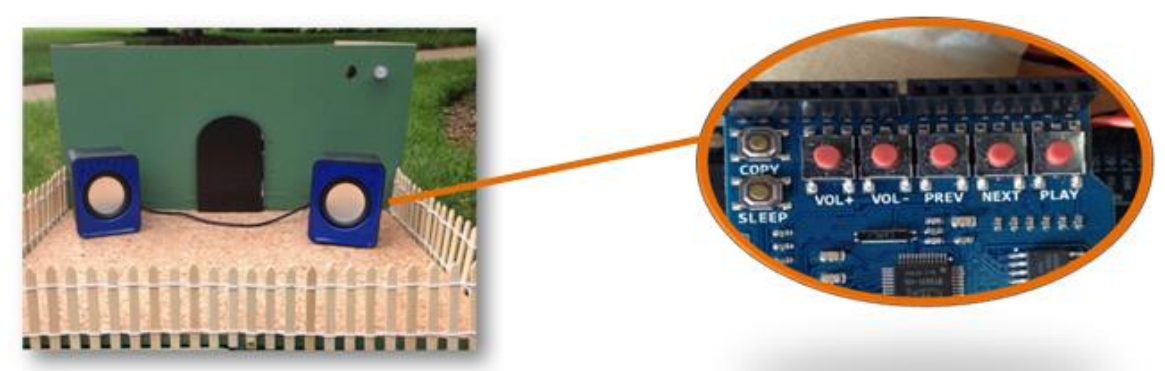

Figure 15. Local Control of the Music Subsystem

The garage door can be controlled remotely. The user has to access the web GUI either from the Android/iOS device or computer and use the DoorOpen and DoorClose action buttons to operate the garage door, as presented in Figure 16.

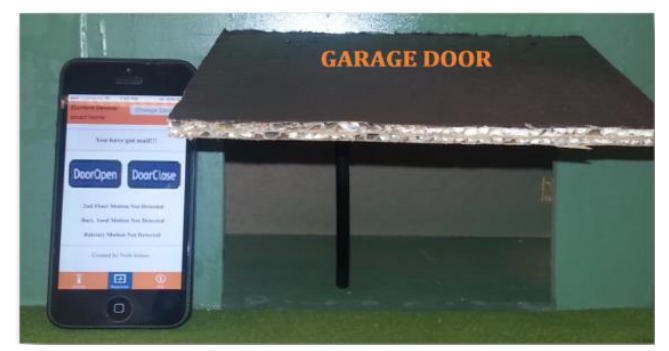

\section{Figure 16. Control of the Garage Door}

\section{Hardware and Software Implementation}

The prototype of the smart house system is presented in Figure 17 and the hardware modules are presented in Figure 18. The system uses an Arduino platform which contains an Arduino Mega 2560 microcontroller, an Arduino Ethernet Shield and an Arduino MP3 Shield. The Arduino Mega 2560 microcontroller is the main controller which performs the control of the functions of the house. All the devices in the house are directly connected to the main controller. The user can access all the functions of the house either from the keypad which is located outside the house or from any device supported by the Android Operating System and the iPhone Operating System (iOS) connected to the Internet.

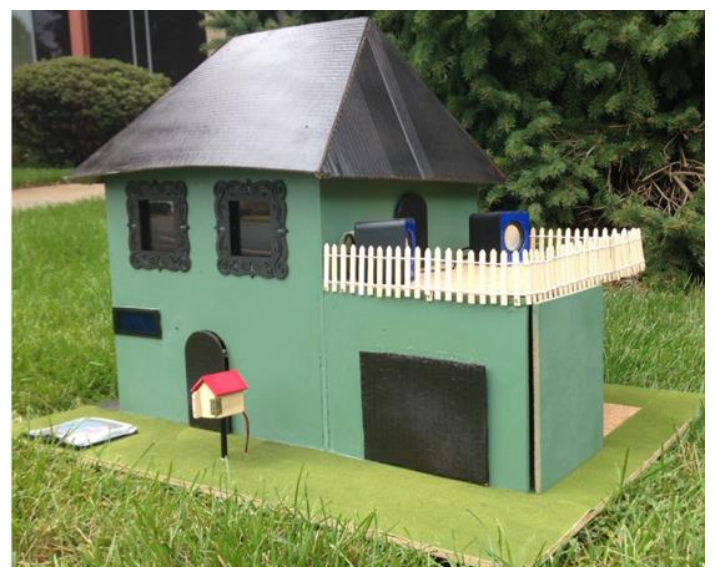

Figure 17. Smart House Prototype

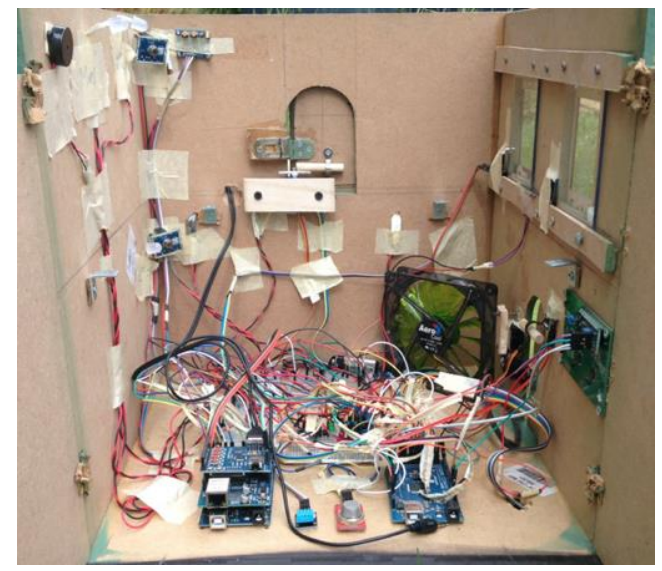

Figure 18. Hardware Modules 
The Arduino Ethernet Shield, which is mounted on top of the Arduino Mega 2560 microcontroller, allows the microcontroller to connect to the internet. This shield comes with a Unique MAC address and it must be assigned a static IP address, a default gateway and a subnet mask. An Ethernet cable is used to connect the Arduino Ethernet Shield to the router, so the router can act as a DHCP server and assign the static IP address. The Arduino Ethernet Shield's associated static IP address is the Universal Resource Locator to the web-server where the user can browse the menu to access all the functions of the house, as presented in Figure 9. Another way to access the menu is to use an open source smartphone application called "Arduino Ethernet Controller" [21]. The Arduino Ethernet Shield with an internet connection allows the Arduino microcontroller to send and receive data from anywhere in the world, as presented in Figure 19.

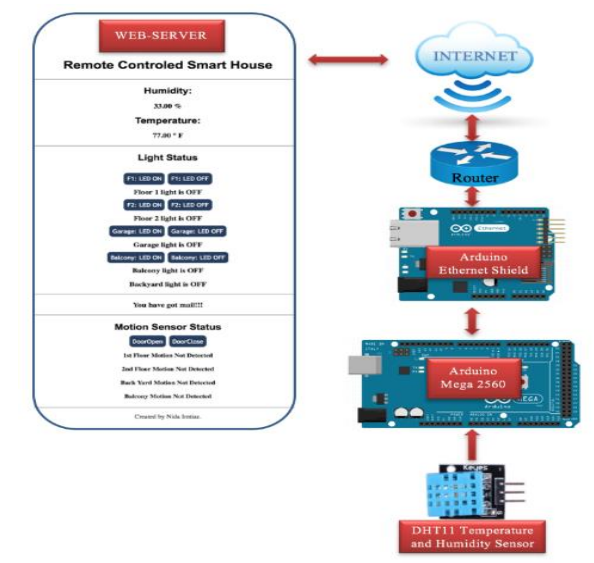

Figure 19. Connectivity between the Web-server and the Microcontroller

\subsection{Energy Management Function Implementation}

The light control subsystem is implemented using an Arduino Ethernet Shield that is connected to the Arduino Mega 2560 microcontroller, several LEDs (Light Emitting Diode) and several PIR (Passive Infra-Red) motion sensors. The hardware module is presented in Figure 20.

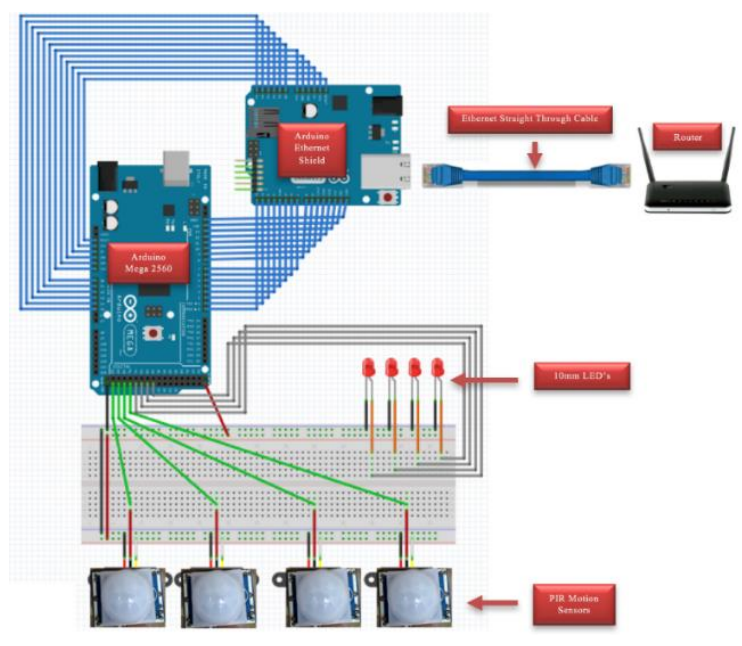

Figure 20. Light Control Subsystem Hardware

To manage the energy consumption, the microcontroller checks the light status and compare it with the PIR motion sensor's status. When a light is on and no movement is detected, the microcontroller will trigger an internal counter. If no movement is detected 
after 5 minutes, the light will turn off. If a movement is detected during the first 5 minutes, the counter will be cleared automatically. This operation can be performed for each light that is on. Figure 21 presents the flowchart of the light control function.

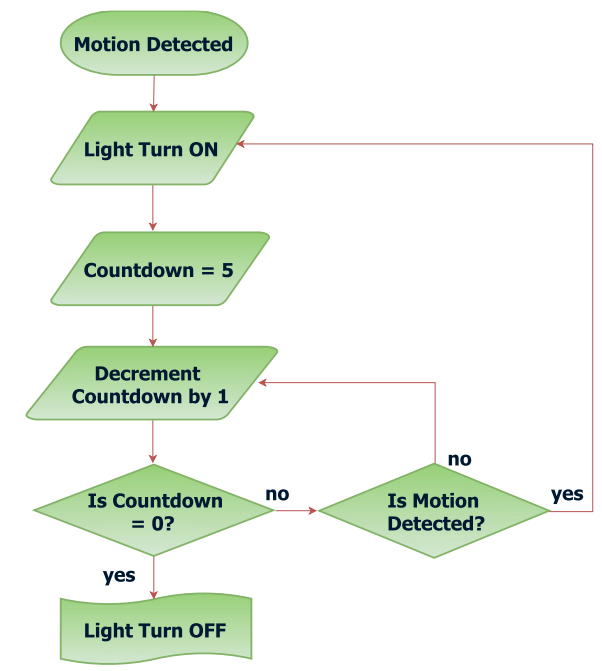

Figure 21. Flowchart of Light Control Function

To remotely monitor and control the lights, the web-server interface is programmed and is loaded into the Arduino Ethernet Shield. It transmits information over the Internet and creates a remote access to the lights in the house. The Arduino Ethernet Shield acts as a server that collects information about the lights' status from the microcontroller and displays that information on the web-server. To gain access to the web-server, the user has to download the free application called "Arduino Ethernet Controller" or use the Arduino Ethernet Shield's IP address as a URL. To remotely turn on or off any light, the user has to select the appropriate action button as presented previously in Figure 9.

The air conditioning subsystem is presented in Figure 22. This subsystem uses the Arduino microcontroller, a DHT11 temperature and humidity sensor, two limit switches, a $1 \mathrm{k} \Omega$ resistor, a NPN transistor, a diode, a $10 \mu \mathrm{F}$ capacitor, a 12-volt battery and a fan to control the house's temperature by adjusting the fan's speed. To maintain a constant temperature in the house this subsystem controls the fan's blade speed (rotation/revolutions) based on the temperature sensor's value. The fan's speed will increase if the temperature increases and vice versa.

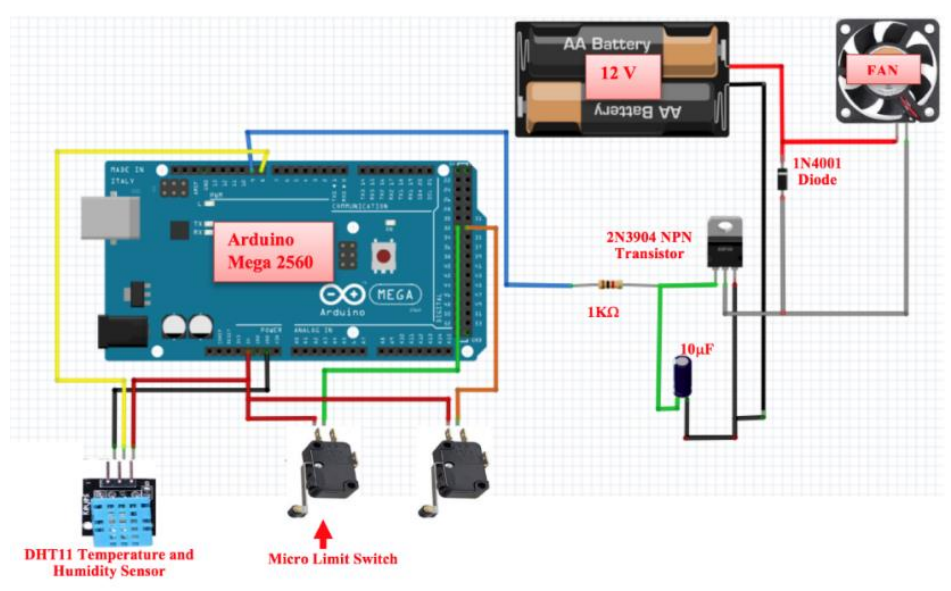

Figure 22. Air Conditioning Subsystem Hardware 
A program was written for the Arduino microcontroller to manage the temperature and the blade rotation speed. The minimum, maximum, and constant (desired) temperature have been defined in the program to determine the minimum and maximum speed of the fan. The desired and the maximum temperature values have been set according to recommended values for a real life air conditioning system. Therefore, the value of the desired temperature is set to $78{ }^{\circ} \mathrm{F}$. The maximum temperature value is set to $110^{\circ} \mathrm{F}$. It was chosen because is the maximum temperature the sensor can withstand and also to avoid dangerous conditions for the inhabitants. When this temperature is reached, an alarm is triggered.

Three conditions have been defined in the structure of the program that maintains a desired temperature in the house. The flowchart for the air conditioning function is presented in Figure 23. The first condition will stop providing electrical power to the fan if the value of the current temperature is less than the minimum temperature. The second condition will increase the power provided to the fan if the value of the current temperature is greater than the desired temperature. The third conduction will turn on an alarm located inside the house, if the current temperature is higher than the maximum temperature.

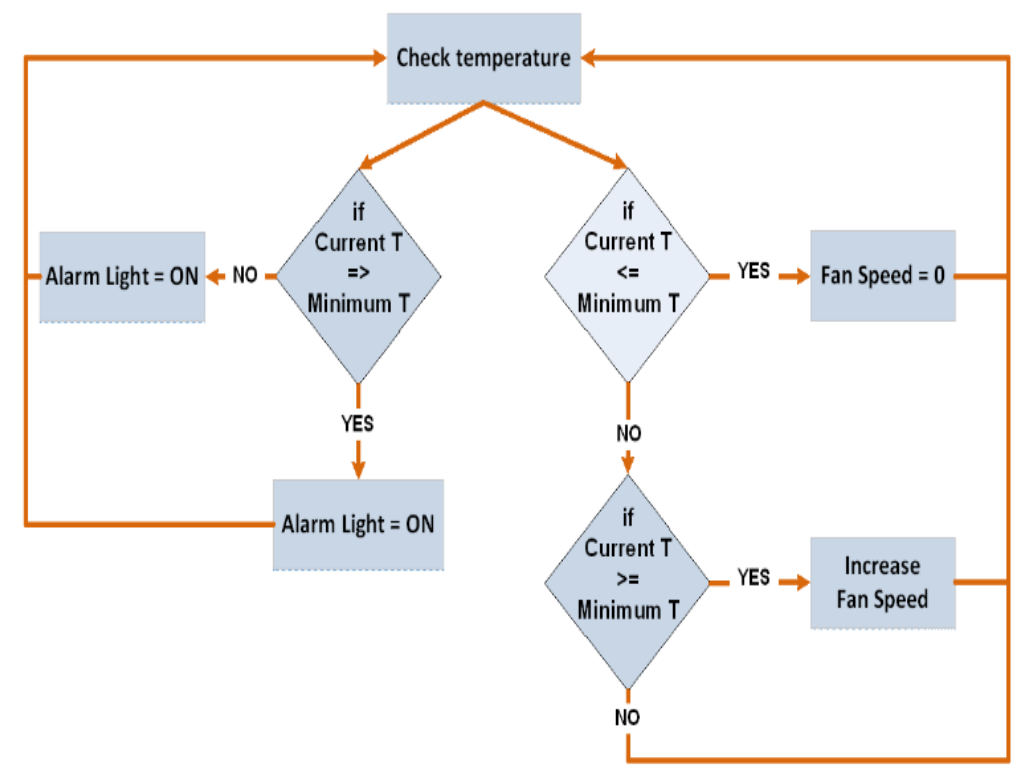

Figure 23. Flowchart of the Air Conditioning Function

To minimize the energy consumption, the fan's speed is decreased by $50 \%$ if a window is detected open. To check if a window is open or closed, limit switches have been installed at the windows. Therefore, if the status of the limit switch is detected high, then the fan's speed will drop by $50 \%$.

\subsection{Security Function Implementation}

The flowchart of the security function is presented in Figure 24 showing how the security levels can be accessed and how the system is designed to monitor the functions of the house locally. 


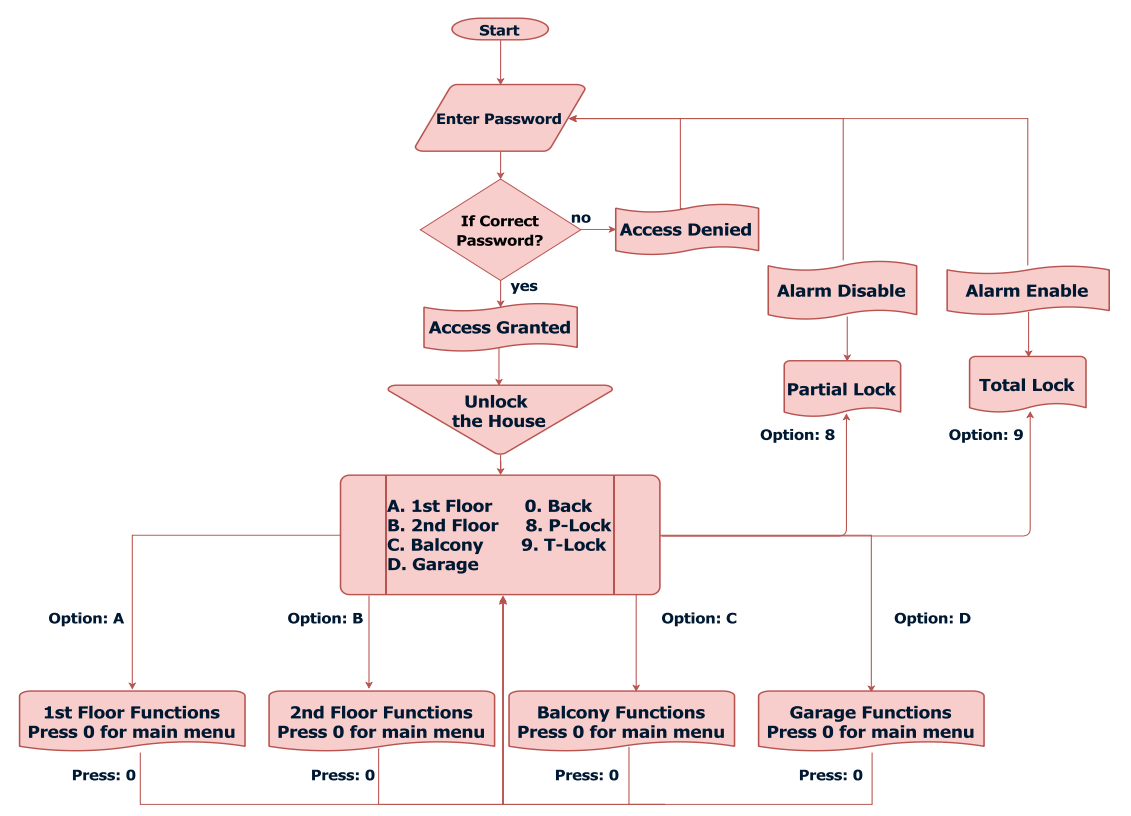

Figure 24. Flowchart of the Security Function

\subsection{Safety Function Implementation}

The safety function is implemented using aMQ-5 gas sensor that detects carbon monoxide $(\mathrm{CO})$, hydrogen $\left(\mathrm{H}_{2}\right)$, liquefied petroleum gas (LPG), methane $\left(\mathrm{CH}_{4}\right)$ and alcohol. The MQ-5 gas sensor is highly sensitive and responds very fast to the system. Its sensitivity level can be adjusted by using the potentiometer located in the front of the MQ-5 gas sensor as presented in Figure 25. The sensor has a sensitive filament inside which has low electrical conductivity in the presence of clean air. When a toxic gas is detected, the electrical conductivity of the filament rises, sending a high input signal to the Arduino Mega 2560, which triggers the alarm inside the house.

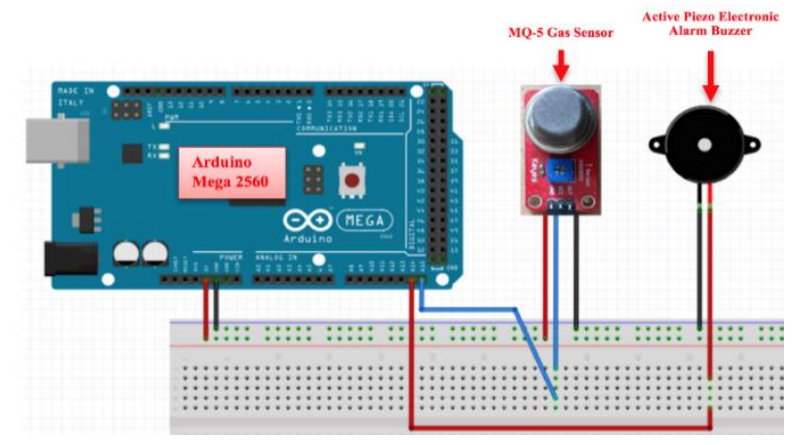

Figure 25. Safety Subsystem Hardware

\subsection{Increased User's Comfort Function Implementation}

The mailbox subsystem uses a limit switch, that contains an actuator that is linked to a set of contacts. When the door of the mailbox is closed, the limit switch makes an electrical connection and when the door of the mailbox is open, the limit switch breaks the electrical connection. Based on the status of the limit switch, the microcontroller turns on and off the "Mailbox Light" located inside the house. The flowchart of the Mailbox Function is presented in Figure 26. Using the Arduino Ethernet Shield, the notification is also sent to the Web-Server saying that "You have got mail!!!" when the mailbox door is open. 
The backyard lighting subsystem has three devices working together: a PIR motion sensor, a photoresistor sensor and 10mm LED. the Arduino Mega 2560 is continuously reading the output signals of the PIR motion sensor and the photo-resistor sensor. The Arduino Mega 2560 sends a high signal to the backyard LED only when it receives a high signal from the PIR motion sensor and the photoresistor sensor at the same time. The flowchart representing the backyard lighting function is presented in Figure 27.

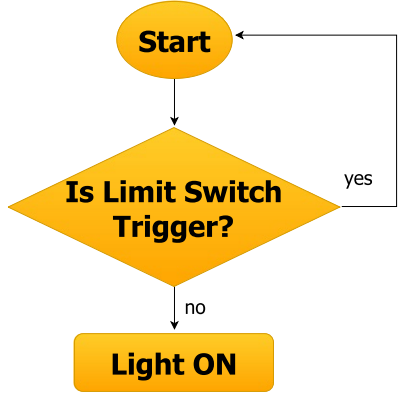

Light ON

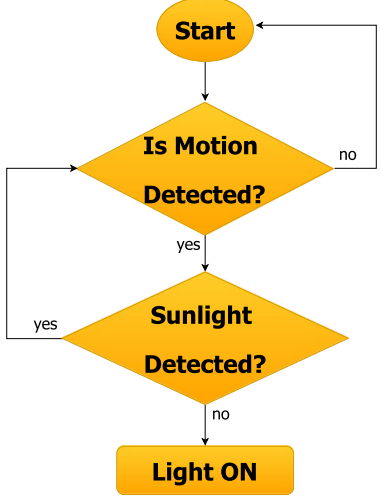

Light ON

\section{Figure 26. Flowchart of Mailbox Figure 27. Flowchart of Backyard Lighting}

The music subsystem is controlled manually using the Arduino MP3 shield that is mounted on top of the Arduino Mega 2560, as presented in figure 5. The Arduino MP3 Shield has a VS1053B Audio Codec Integrated Circuit which can decode a wide variety of audio formats such as MP3, WMA, AAC and WAV. This shield also has SD Socket that is being used by the Arduino SD library to read music files off the SD card and stream them to the VS1053B. In order to drive the audio out of the Arduino MP3 shield, the on-board $3.5 \mathrm{~mm}$ stereo audio jack is connected to the $12 \mathrm{~V}$ speakers.

The garage door subsystem uses the SG90 servo motor. SG90 servo motors are connected to all the doors of the house, including the garage door as presented in Figure 28. The servomotor can rotate $180^{\circ}\left(90^{\circ}\right.$ in each direction). Using the smartphone app, when the user opens the garage door, a signal is sent to the Arduino Ethernet Shield. The signal is then passed to the microcontroller allowing the SG90 servo motor to rotate $90^{\circ}$ outside (to the left) and open the door. A similar process occurs to close the garage door. The user can change the position of the door so it can open more than $90^{\circ}$ or less than $90^{\circ}$ by modifying the program dedicated to the garage door.

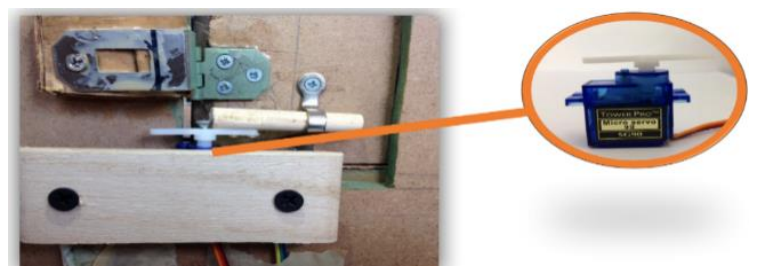

Figure 28. SG90 Servo Motor

\section{Tests and Results}

After the system was implemented, extensive testing was performed, in order to have a full evaluation of the correct operation of the system and to prove its feasibility, efficiency, reliability, and ease of use. 


\subsection{Test of the Energy Management Subsystem}

The light control subsystem was tested by moving the hand on each floor and observing the light turning on as shown in Figure 29. It was also tested using the smart phone application and the web-server by turning on and off all the lights in the house as presented in Figure 29. The subsystem successfully passed all the tests.
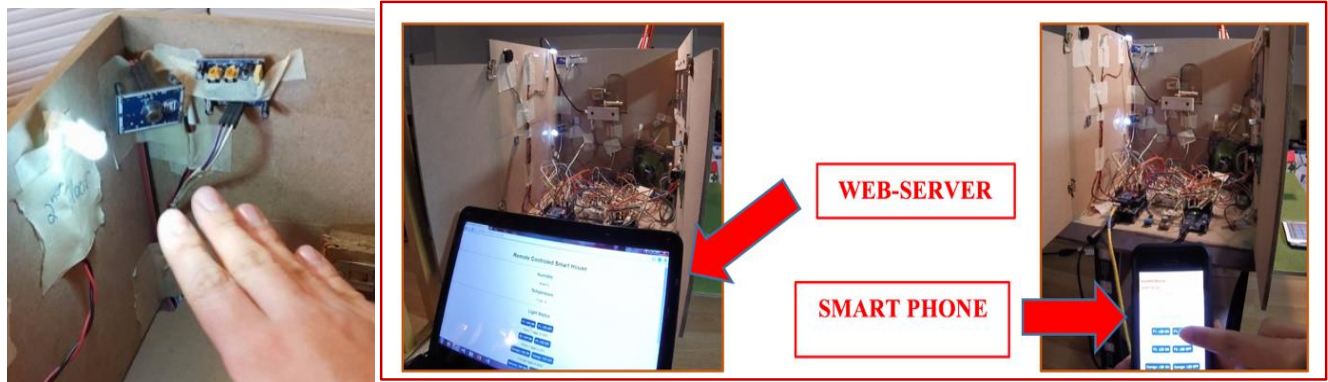

Figure 29. Testing the Light Control Subsystem Manually and Remotely

The air conditioning subsystem was tested by observing the fan's speed while the temperature was changing inside the house. In order to test the fan's speed, a lighter was used to increase the temperature in the house. As expected, the fan's speed gradually increased as presented in Figure 30.
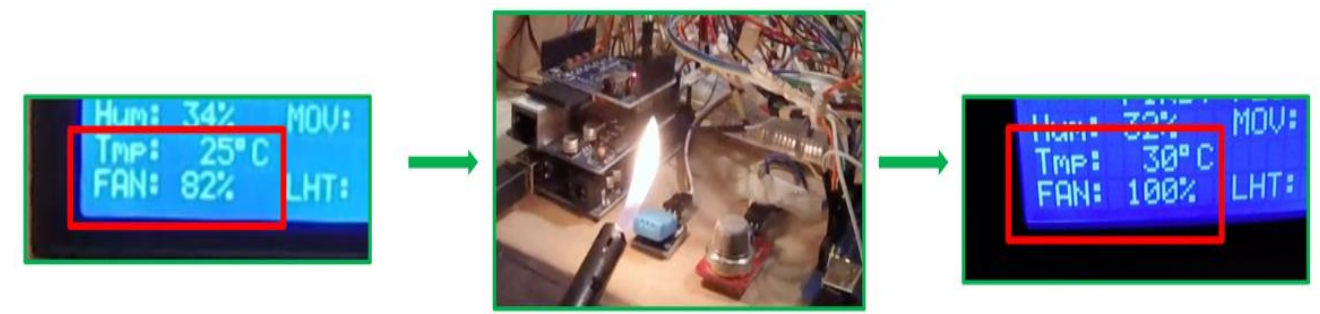

Figure 30. Testing the Fan's Speed Using a Lighter

To test the connection between the fan's speed and the windows' status, the windows were closed and opened, and the fan's speed was monitored as presented in Figure 31.

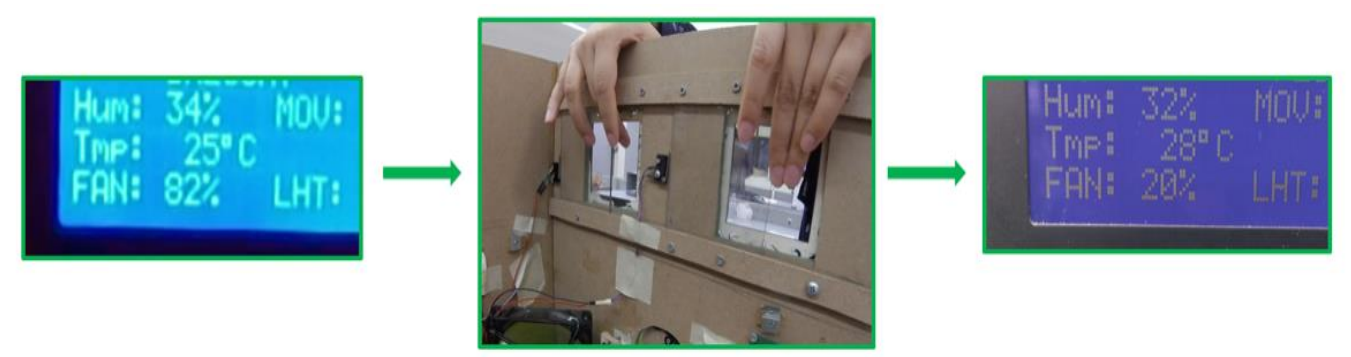

Figure 31. Testing the Fan's Speed by Opening the Windows

\subsection{Test of the Security Subsystem}

All the security levels of the house were tested intensively by using the keypad and the LCD screen. To test the "Unlocked" mode, an incorrect pin was entered and as expected the red LED turn on and the LCD screen prompt "ACCESS DENIED! INVALID CODE" as presented in Figure 10. When the correct pin was used all the doors opened simultaneously, the green LED turned on and the LCD screen prompt "ACCESS GRANTED WELLCOME!!". The "Partially Locked" and "Total Locked" modes were 
tested using the menu of the house displayed on the LCD screen as presented in Figure 10. When the number 8 on the keypad was pressed to go into "Partially Locked" mode, the hand was moved on each floor for the PIR motion sensors to detect the movement. The subsystem gets the expected result because the LCD screen displays the movement detected in each floor but the alarm is not triggered. The same action is performed in the "Total Locked" mode and as expected, the LCD screen displays the movement detected in each floor and triggers the alarm.

\subsection{Test of the Safety Subsystem}

A small lighter can be used to successfully test the safety subsystem. When the gas is released from the lighter and is detected by the MQ-5 gas sensor located inside the house, the alarm is triggered, as presented in Figure 32.

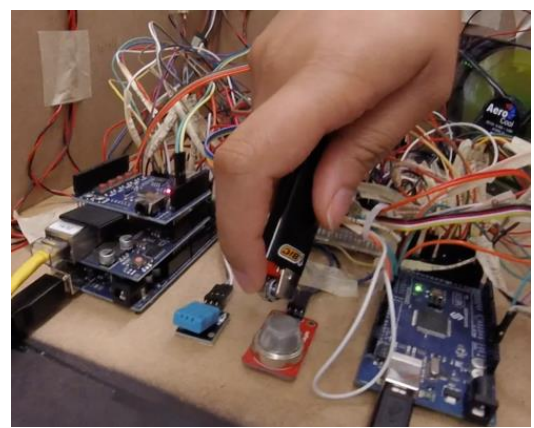

Figure 32. Testing the Safety Subsystem

\subsection{Test of the Increased User's Comfort Subsystem}

The mailbox subsystem was tested by frequently opening and closing the mailbox door and monitoring the mailbox light turning on inside the house along with the message prompted on the web-server saying "You have got mail!!!".

The backyard light subsystem turns on the light only when movement is detected and there is not sunlight. The hand was moved in front of the PIR motion sensor and the photoresistor sensor was covered temporarily to block the sunlight. Under these action, the light turned on. When the photoresistor sensor was not covered, the backyard light turned off. The testing of this subsystem is presented in Figure 33.

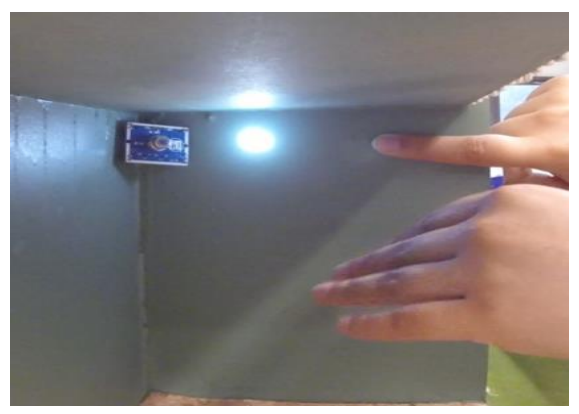

\section{Figure 33. Testing the Backyard Light Subsystem}

The music subsystem was tested by inserting the SD card inside the Arduino MP3 Shield's SD slot and playing all the music files from it. The testing also includes skipping songs, adjusting the volume, forwarding and reversing songs, pausing and copying the current song onto the Arduino MP3 Shield's flash memory.

The garage door subsystem was tested using the smart phone application and the Internet browser on the laptop. When the action button "DoorOpen" was pressed from the 
smart phone or Internet browser, the garage door opened in three seconds and when the action button "DoorClose" was pressed, the garage door closed in three seconds, as presented in Figure 16. These three seconds represent the time required to process the data between the web-server and the garage door's servo-motor.

\section{Reliability Analysis}

CARE-Computer Aided Reliability Engineering software tool was used to predict the reliability parameters of the smart house system. CARE is an engineering tool, developed by BQR Reliability Ltd., intended for reliability analyses and testing of electronic and mechanical systems [22]. The Reliability $R(t)$ of a system follows an exponential distribution law during the useful life phase of the system:

$\mathrm{R}(\mathrm{t})=\mathrm{e}^{-\lambda \mathrm{t}}$

where $\lambda$ represents the failure rate and is assumed to have a constant value during the useful life of the system and $t$ represents time [23].

The Mean Time to Failure (MTTF) of a system is the expected time of the occurrence of the first system's failure. If the reliability function is defined by equation (1), then:

$\mathrm{MTTF}=1 / \lambda$

The estimation of the failure rate $\lambda$ of electronic components and systems are performed using military or commercial standards. The reliability estimations presented in this paper are based on the Mil-Std-217-US DOD standard/ handbook [24].

Using the schematics (hardware modules) of the system, the serial reliability model was created in CARE MTBF module. The serial system assumes that all the components should survive for the system to operate correctly. The reliability of the system, assuming exponential failure rate for each component, is given by the following expression:

$$
R_{\text {series }}(t)=e^{-\sum_{i=1}^{n} \lambda_{i} t}=e^{-\lambda_{\text {systent }} t}
$$

Figure 34 presents the product tree (serial model) for reliability analysis. Figure 35 presents the product tree and the list of conditions (prediction method, temperature, environment, component's quality, etc.) for reliability analysis. The prediction method is S217F2, the environment-Ground Benign and the quality of the components-low (commercial grade components). The results of the analysis are presented in Figure 36.

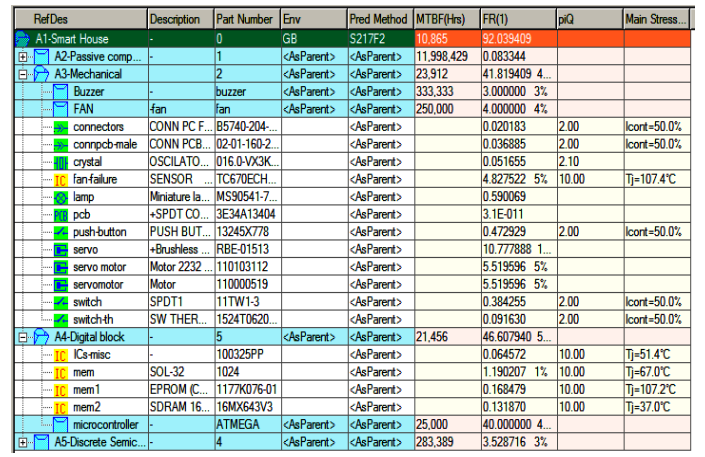

Figure 34. Reliability Serial Model for the Smart House System 


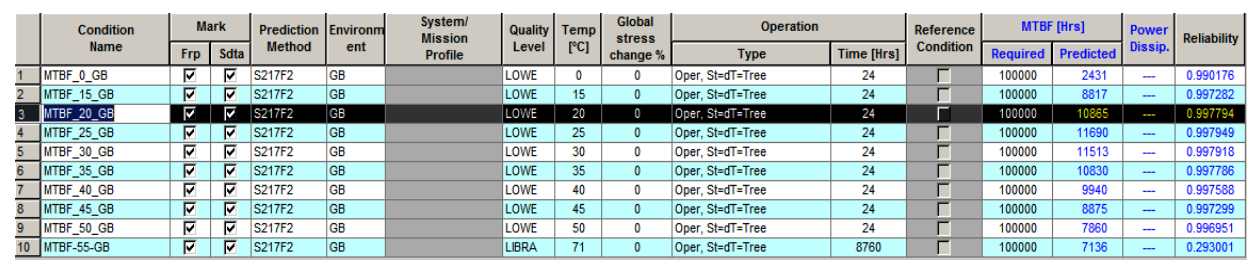

Figure 35. List of Conditions for Reliability Analysis
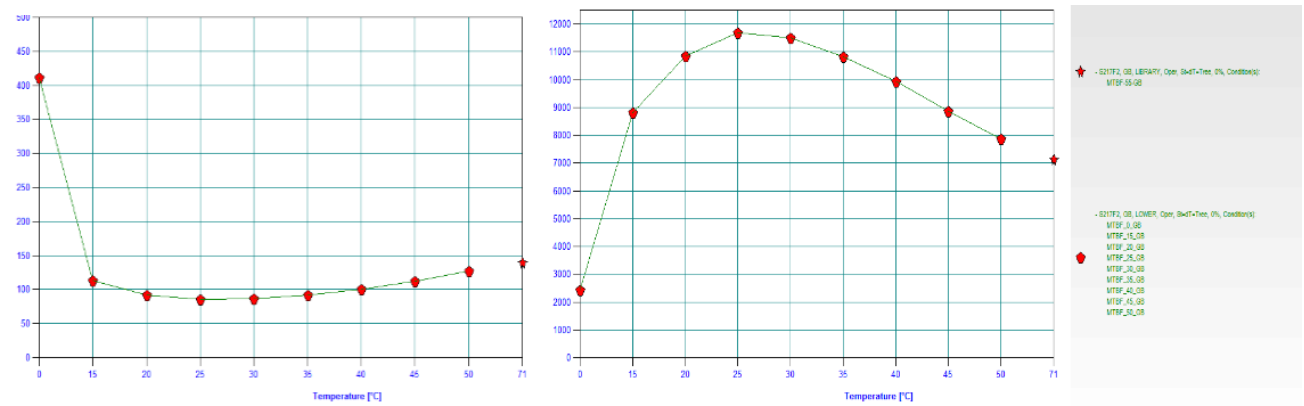

Figure 36. Failure Rate and MTTF Estimation versus Temperature

The failure rate of the system (and MTTF accordingly) is temperature dependent using the S217F2 Full Stress prediction method. The higest failure is at the ambient temperature $0^{\mathrm{O}}$ Celsius and the lowest around $25^{\circ}$ Celsius as expected, for the temperature range considered for this analysis. The Pareto distribution of failure rates of various components is presented in Figure 37. The highest contribution to the failure rate of the entire system is given by the family of integrated circuits (large number of ICs in the system), followed by mechanical components. The category "miscellaneous" includes components that were not identified in the CARE software library and their failure rate was estimated by the user, using Mil-Std-217-US DOD handbook.

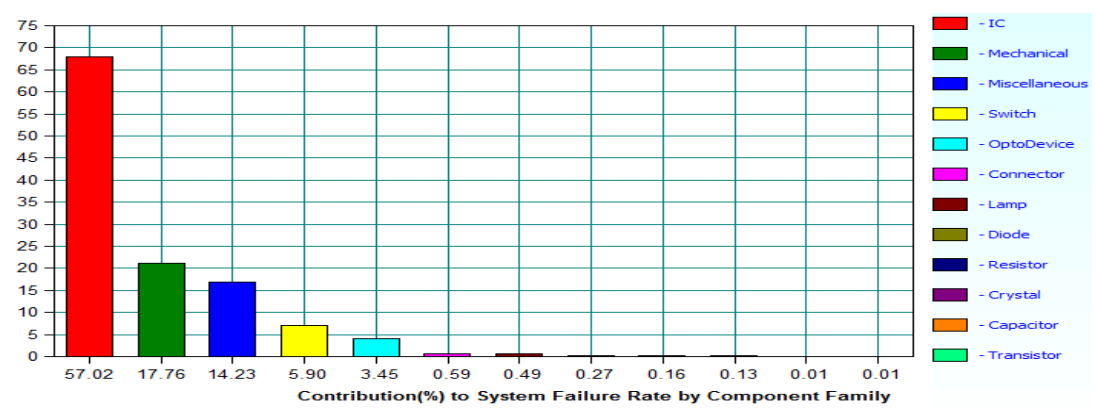

Figure 37. Failure Rate by Component's Family

Results of complex reliability estimations of the system are presented in [25]. Estimating the reliability of various hardware blocks of the system and identifying components based on their failure rates, helps the designer to make the necessary changes to the design, choosing more reliable components, incorporating spares or adding faulttolerant features.

\section{Conclusions and Future Work}

The design and implementation of a reliable and environmental-friendly smart home was presented. The system is affordable, user friendly and easy to adapt, allowing to add 
new functions, without altering previously built functions. All the functions of the smart house were tested and are fully functional. The prototype of smart house is equipped with solar panels, adding more value to the environmental friendly house concept. Future and present work includes how to design and implement assistive technological solutions for vulnerable people. Challenges include how to transfer the needs of an individual to specific "smart devices" in a reliable manner and install them in a way that will achieve their intended purpose.

The smart house project is currently used for live demonstration emulating customerowned equipment during academic open houses and to raise public awareness regarding the advantages of a smart house as part of the mission of the Renewable Energy and Sustainability Center at Farmingdale State College, New York.

\section{Acknowledgments}

The authors gratefully acknowledge the support of the Provost Office, RESC Center, and C-Step Program at Farmingdale State College and BQR Reliability Engineering Ltd.

\section{References}

[1] S. Kumar, "Ubiquituos Smart Home System Using Android Application", International Journal of Computer Networks\& Communications, vol. 6, no. 1, (2014), pp. 33-43.

[2] G. Jenkins, "The Internet of Things: Reliability and Trust Issues", Available at: http:// my_weblog/2016/01/reliability-smart-devices.html, (2016).

[3] R. Panna and R. Thesrumluk, "Development of Energy Saving Smart Home Prototype", International Journal of Smart Home, vol. 1, no. 1, (2013), pp. 47-62.

[4] L. Wang, D. Peng and T. Zhang "Design of Smart Home System Based on WIFI Smart Plug", International Journal of Smart Home, vol. 9, no. 6, (2015), pp. 173-182.

[5] S. R. M. Zeebaree and H. M. Yasin, "Arduino Based Remote Controlling for Home: Power Saving, Security and Protection", International Journal of Scientific \&Engineering Research, vol. 5, no. 8, (2014), pp. 266-272.

[6] R. Mafrur, K. Bang and D. K. Lee, "Developing and Evaluating Mobile Sensing for Smart Home Control", International Journal of Smart Home, vol. 9, no. 3, (2015), pp. 215-230.

[7] Y-C. Yu, S. D. You and D-R. Tsai, "Smart Door: A Ubiquitous Collaboration System for Home Activities in the Smart Home", Journal of Information Science and Engineering, vol. 2, (2013), pp. $1227-1248$

[8] J. Bangali and A. Shaligram, "Design and Implementationof Security Systems for Smart Home based on GSM Technology”, International Journal of Smart Home, vol. 7, no. 6, (2013), pp. 201-208.

[9] R. Hassan and M. Khan, "Microcontroller Based Home Security System with GSM Technology", Open Journal of Safety Science and Technology, vol. 5, (2015), pp. 55-62.

[10] D. K. Paraskumar, A. Pandey, P. Kimar and D. Javale, "Home Security System", International Journal of Inventive Engineering and Sciences, vol. 2, no. 12, (2014), pp. xx.

[11] L. Kamelia, A. Noorhassan, S. R. M. Sanjaya and W. S Edi Mulyana, "Door-Automation System Using Blue Tooth-Base Android For Mobile Phone", APRN Journal of Engineering and Applied Sciences, vol. 9, no. 10 (2014), pp. 1759-1762.

[12] S. Sedhumadhavan and B. Saralevici, "Optimized Locking and Unlocking a System Using Arduino", International Journal of Innovative Research in Computer and Communication Engineering, vol. 2, no. 11, (2014), pp. 6658-6663.

[13] A. Fahmi, E. Kodyrov, A. D. Choi and G. Lee, "Hey Home, Open Your Door, I'm Back! Authentication System using Ear Biometrics for Smart Home", International Journal of Smart Home, vol. 7, no. 1, (2013), pp. 173-181.

[14] W. H. Wan Ismail, H. R. M. Husny and N. Y. Abdullah, "Smoke Detection Alert System via Mobile Application", Proceedings of the 10th International Conference on Ubiquitous Information Management and Communication, Danang, Vietnam (2016) January 04-06.

[15] S. P. Panchangan and P. Naikan,"Failure Analysis Methods for Reliability Improvement of Electronic Sensors", International Journal of Recent Technology and Engineering, vol. 1, no. 3, (2012), pp .83-86.

[16] P. Panchangan and P. Naikan, "Reliability Analysis of Temperature Sensor System", International Journal of Reliability, Quality and Safety Engineering, vol. 20, no.1, (2013), pp. 1350003-1-1350003-20.

[17] Dewsbury G. and M. Edge, "Designing the Home to Meet the Needs of Tomorrow;; Today: Deconstructing and Rebuilding the Home for Life"', Proceeeding of European Network for Housing Research, Gavle, Sweden, (2000), June 26-30.

[18] Dewsbury G. and M. Edge, "Designing the Home to meet the Needs of Tomorrow", Open House International, vol. 26 , no. 2 , (2001), pp. 33-42. 
[19] Dewsbury G. and M. Edge, "Designing Safe Smart Home Systems For Vulnerable People”, Proceedings of the Dependability and Healthcare Informatics Workshop, Edinburgh, UK, (2001), March 10-12.

[20] M. Ferrantino, "Arduino Ethernet Controller Support" , Available at: https://itunes.apple.com/us/app/arduino-ethernet-controller, (2015).

[21] Arduino Language Reference, Available at https://www.arduino.cc/en/Reference (2016).

[22] BQR-CARE-8-RBD-V8.8 User Manual, Available at: https://www.bqr.com (2015).

[23] D.Sieworek, R. Swarz,"Reliable Computer Systems-Design and Evaluation"A.K. Peters, Ltd, (1998).

[24] Military Handbook for "Reliability Prediction of Electronic Equipment"- MIL-HDBK-217, Available at: http://www.sre.org/pubs (1991).

[25] M. Radu," Reliability Analysis of Smart House System", indexed by IEEE Explorer, IEEE International Energy and Sustainaibility Conference, Koln, Germany, (2016), June 28-30.

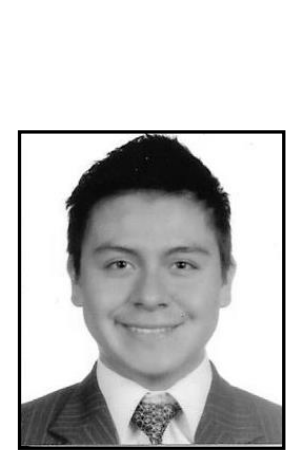

\begin{abstract}
Authors
Diego Paucar, he received a BS degree in Electrical Engineering Technology with a dual major in Computer Engineering Technology from Farmingdale State College, Farmingdale, NY in 2015. Diego is currently working as project manager at Broadband Office Solutions, New York, USA. His areas of research interest are SIP Trunking, Hosted PBX, Internet Access Bonding, and Cloud-based Network Administration and Home Automation.
\end{abstract}

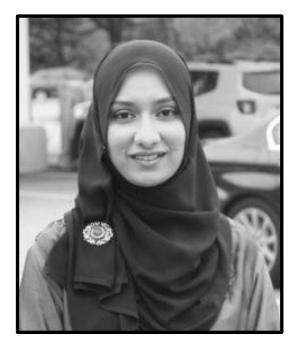

Nida Imtiaz, she received a BS degree in Electrical Engineering Technology with a dual major in Computer Engineering Technology from Farmingdale State College, Farmingdale, NY in 2016. Nida is presently working as Network Administrator for Nasdaq, Philadelphia, USA. Her research areas of interest are Microcontroller, Internet of Things, Home Automation and Wireless Sensor Networks.

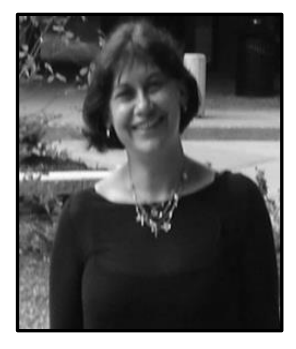

Mihaela Radu, she received a Ph.D. in Electrical Engineering from the Technical University of Cluj-Napoca, Romania, in 2000. She is currently a faculty member at Farmingdale State College, NY, USA. Her current research interests include Reliability and Fault Tolerance of Electronic Systems, Testing of Digital Systems, Digital Design using Programmable Logic Devices. She is member of IEEE and ASEE societies and research associate for the Renewable Energy and Sustainability Center. 
International Journal of Smart Home

Vol. 11, No. 1 (2017) 